\title{
Accommodation to uncomfortable patterns
}

\author{
Sarah M Haigh ${ }^{1}$, Wolfgang Jaschinski ${ }^{2}$, Peter M Allen ${ }^{3}$, Arnold J Wilkins ${ }^{1} \S$ \\ ${ }^{1}$ Visual Perception Unit, Department of Psychology, University of Essex, Wivenhoe Park, \\ Colchester, Essex CO4 3SQ, UK; e-mail: arnold@essex.ac.uk; ${ }^{2}$ Leibniz-Institute für \\ Arbeitsforschung an derTU Dortmund, Ardeystrasse 67, D-44139 Dortmund, Germany; \\ ${ }^{3}$ Vision and Eye Research Unit, Postgraduate Medical Institute, Anglia Ruskin University, \\ Cambridge CB1 1PT, UK \\ Received 19 October 2012, in revised form 13 February 2013
}

\begin{abstract}
Grating patterns can cause discomfort and perceptual distortions. Individuals who experience discomfort and are susceptible to these distortions generally show weaker accommodation than those who are less susceptible. We measured the accommodative response to grating patterns known to differ in the discomfort they evoke because of differences in their colour, motion, or spatial frequency. The parameters known to affect discomfort and distortion had no influence on the mean or variance in the accommodative response, even when accommodative demand was manipulated systematically and the accommodative response varied as expected.
\end{abstract}

Keywords: accommodation, pattern sensitivity, gratings

\section{Introduction}

In patients with photosensitive epilepsy, patterns of stripes can evoke paroxysmal epileptiform electroencephalographic activity. The patterns to which patients are susceptible are high in contrast and have a spatial frequency between 0.5 and 12 cycles deg $^{-1}$ (cpd), and susceptibility is maximal at about $3 \mathrm{cpd}$. Such patterns are generally perceived as uncomfortable to view by healthy individuals and can evoke visual illusions. Some individuals are more susceptible to the illusions than others, and these individuals are generally more prone to headaches (Wilkins et al 1984). Illusions are sometimes reported when reading text, and the illusions seen in text can resemble those seen in gratings: a rhomboid lattice, rainbow colours, and movement (Wilkins and Nimmo-Smith 1987). Some aspects of the perceptual instability have been explained as due to accommodative fluctuations (Campbell and Robson 1958; Helmholtz 1856/1924; Millodot 1968).

Accommodative fluctuations have also been proposed as an explanation for a variety of visual illusions, including the distortions seen in fine grating patterns (Helmholtz 1856/1924), concentric circles (Helmholtz 1856/1924; Purkinje 1823, 1825), and in the 'fluttering hearts' illusion. Purkinje argued that the distortions (specifically in concentric circles) were due to an unfocused image on the retina, but it was Helmholtz (1856/1924) who specifically identified the accommodative system as being potentially responsible for the blurred and distorted image. Brewster, Wheatstone, and Helmholtz (Wade 1983) observed that a red shape (in this case a heart shape) on a green background created an illusion of depth in which the background and the shape appeared to oscillate, causing a 'fluttering' illusion. There has been much speculation why this illusion might occur. One possibility is that, owing to longitudinal chromatic aberration of the eye, the normal fluctuations in accommodation become detectable. Another possibility is that the accommodative fluctuations are exaggerated because the accommodative system is "colour blind" (Wolfe and Owens 1981) and cannot detect the contours of the shape from the background, particularly when the contour is isoluminant.

$\S$ Corresponding author. 
Isoluminance has an effect on form perception (Gregory 1977), despite the isoluminant chromatic contours being well above detection threshold (Switkes et al 1990).

In the majority of studies that have measured the accommodative response to isoluminant patterns, the variance in the response has not been reported. Switkes et al (1990) found that the accommodative response to isoluminant contours was much weaker compared to contours with high luminance contrast. Wolfe and Owens (1981) similarly found a poor accommodative response to isoluminant contours compared to contours with high luminance contrast. They did not record accommodation continuously, but remarked that they did not see any great fluctuations in the accommodative response over time.

The difference between the accommodative response and accommodative stimulus appropriate for the actual viewing distance is known as the accommodative lag and varies from one individual to another: the unit of these measures is the dioptre, the reciprocal of focal distance in metres. Individuals with large accommodative lags tend to report discomfort and illusions. Allen et al (2010) measured the effect of an achromatic $1.3 \mathrm{cpd}$ grating pattern on accommodative lag in participants who reported seeing a large number of illusions in a pattern of stripes. They found that these participants had a larger accommodative lag (they under-accommodated for the viewing distance) than those who reported few illusions. When the participants used a tinted overlay of their choice, the lag and the discomfort were both reduced. Simmers et al (2001) also found that tinted lenses reduced the variability in the accommodative response in those who reported discomfort and illusions. Therefore, it is possible that it is the weaker accommodative response (and the consequent blur) that causes the illusions and discomfort.

Similarly, Chase et al (2009) found a positive correlation between the accommodative lag in response to a $0.2 \mathrm{~m}$ target and the visual discomfort scores from the Conlon Visual Discomfort Survey. Tosha et al (2009) also found that a group which reported high discomfort showed greater fatigue when sustaining accommodation at near distance (3 D-4 D), even over a short time period (90 s).

A poor accommodative response does not appear to explain all of the illusions that have previously been reported in stripes. A rhomboid-lattice illusion is often seen (Wilkins 1986). The size of the lattice appears to be affected by the spatial frequency of the target pattern (Wilkins and Nimmo-Smith 1987). It cannot readily be explained by peripheral factors and an explanation in terms of cortical inhibition has been proposed by Georgeson (1976, 1980).

An alternative theory for the cause of the visual discomfort and illusions seen in striped patterns is that the cortex produces a heightened neural response to the pattern (Huang et al 2003). It is possible that the excess firing can spread locally to nearby parts of the cortex and can cause the appearance of illusions. Adjamian et al (2004) found maximal cortical power in the gamma frequencies to patterns which caused the most discomfort and illusions. Siniatchkin et al (2007) also found larger N75-P100 and P100-P135 components in the visual evoked potential in response to uncomfortable patterns in individuals with a propagating photoparoxysmal response but not in those with local photoparoxysmal response. This could explain why some individuals are more susceptible to illusions in grating patterns: those with a hyperexcitable cortex [eg individuals with migraine (Markus and Soso 1989)] produce an over-response to the patterns causing more illusions. It is also possible, therefore, that the weaker accommodative response to the uncomfortable images is a mechanism to reduce the discomfort, rather than a cause of the discomfort.

In the following four experiments, we investigated the differences in the accommodative response to gratings that differed with respect to pattern parameters known to affect how uncomfortable the patterns are to view. The first three studies investigated the accommodative 
lag in response to grating patterns that varied in parameters of colour or movement. In the final study, we varied the accommodative demand, and investigated the differences in accommodative lag in response to grating patterns that varied in their spatial frequency and contrast. If a poor accommodative response is responsible for the discomfort, then one might anticipate an association between the patterns that normally evoke discomfort and the accommodative lag when viewing these patterns.

\section{Experiment 1. Accommodation to coloured gratings}

Chromatic contrast, as well as luminance contrast, is known to contribute to visual discomfort (Haigh et al 2012a, 2012b; Wilkins et al 2008). Wilkins et al (2008) measured the ratings of discomfort to a series of chromatic grating patterns that varied in the separation in chromaticity of the component bars. Participants were asked to rate how uncomfortable the pattern was to view on a Likert scale. The gratings varied in the hue and saturation of the component bars, and in all experiments there was a linear increase in aversion with the separation in CIE UCS chromaticity of the bars, irrespective of their luminance contrast and hue. In principle, it is possible that the large separations in chromaticity could be causing a weak accommodative response and/or large fluctuations in accommodation because of longitudinal chromatic aberration: the accommodative system might attempt to accommodate to two different distributions of spectral power.

In the following experiment, the mean chromaticity of a grating was varied systematically. Three types of grating were presented, each having colours formed from just two of the three colour pixels of an LCD display: ie with chromaticities on the red-green, green-blue, and red-blue extremes of the display gamut. The accommodative response was measured with an open-field autorefractor.

\subsection{Method}

2.1.1 Participants. All studies were approved by the University of Essex Ethics Committee, and adhere to the tenets of the 2008 Declaration of Helsinki. Four male and twenty-six female undergraduate students aged 18-33 years (mean 20 years) from the University of Essex took part. All had a minimum acuity of $6 / 6$ monocularly at near and binocularly at distance and near (Lighthouse Near and Far tests of visual acuity) and a minimum stereoacuity of $60 \mathrm{~s}$ arc (Titmus test). Log contrast sensitivity for letters was at least 2.00 (Pelli-Robson letter chart), and no red-green anomalies were detected (Ishihara plates). None of the participants required prescription glasses or contact lenses.

Participants completed a questionnaire about their general health, visual history, and the headaches they experienced. None of the participants had a history of seizures.

2.1.2 Stimuli. A telespectroradiometer (model PR-670, Photo Research, Chatsworth) was used to measure the $\left(u^{\prime} v^{\prime}\right)$ chromaticity of the red, blue, and green pixels from a Dell Precision M4500 laptop screen. The chromaticities used for the gratings were made up of only two of the red, blue, or green pixels. The chromaticity of the point midway between the chromaticities of the red pixel and the green pixel was found. Pairs of colours (with the same photometric luminance, $23 \mathrm{~cd} \mathrm{~m}^{-2}$ ) that lay either side of the midpoint and equidistant from it were used in alternating bars in a $2 \mathrm{cpd}$ square-wave grating pattern. The colour distance between the paired chromaticities was gradually increased to make seven gratings. For example, the smallest colour difference was between two shades of yellow. The colour difference increased to make a grating with bars of yellow-red and yellow-green, with the largest colour difference being a grating with bars of a red and a green. This was repeated for the red-blue and the blue-green colour pairs to create a total of 21 gratings. The chromaticities of the colours used are shown in figure 1. 


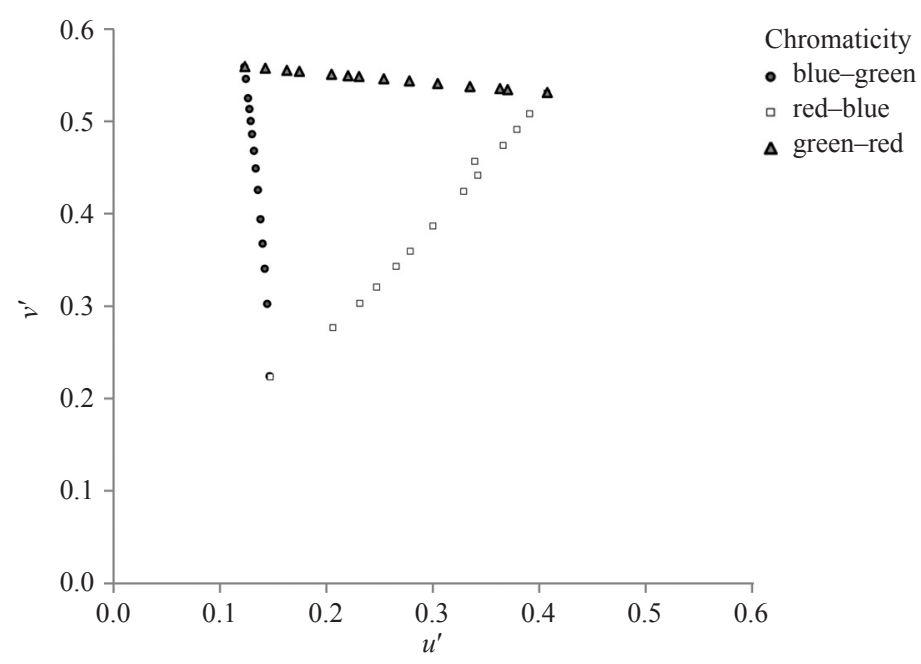

Figure 1. CIE UCS $1976 u^{\prime} v^{\prime}$ diagram of the chromaticities used in experiment 1, shown separately for the three types of grating. The bars were generated initially using measurements from a Minolta Color Analyser, but the chromaticities shown were measured subsequently with a Photoresearch PR-670 telespectroradiometer; hence the slight variation in chromaticity from the linear pattern expected.

The gratings were horizontal, circular in outline, and from the viewing distance of $0.5 \mathrm{~m}$ subtended $20 \mathrm{deg}$. A central fixation saltire cross $(3 \mathrm{~mm})$ was present throughout the entire trial. This was added to provide a stable point for the participant to fixate. Ensuring that the participant was fixating the centre of the grating pattern provided a similar field of view for all patterns for all participants, and reduced eye movements that otherwise would have disturbed the autorefractor recording (Wolffsohn et al 2002).

\subsubsection{Procedure}

Accommodation was measured with the WAM-5500 autorefractor (Grand Seiko, Fukuyama, Japan). The autorefractor recorded the accommodative response and the pupil diameter at $2 \mathrm{~Hz}$ (prorated as necessary to allow for the occasional data dropout). Recordings were taken from the left eye, but the stimuli were viewed binocularly. The 21 gratings were presented in random order, each for $10 \mathrm{~s}$, separated by $3 \mathrm{~s}$ during which a grey screen of similar spaceaveraged luminance was presented. The participant was asked to fixate the central fixation cross throughout the trial. The gratings were presented twice in separate trials, separated by a short break.

2.1.4 Data analysis. At the outset of the recording, a measurement of accommodation at $8 \mathrm{~m}$ was obtained. The spherical value was used to adjust the accommodative response so as to take into account individual refractive error. The mean and standard deviation of participants' accommodative response during the $10 \mathrm{~s}$ stimulus presentations were obtained. The mean pupil diameter during stimulus presentation was also analysed.

\subsection{Results}

Data from three participants were excluded because the autorefractor was unable to obtain a reading for all of the stimuli. Poor recordings were due to excessive blinking and/or a small pupil diameter. The accommodative response was expressed as a lag of accommodation by calculating he difference between the accommodative response and the distance of the target in dioptres. The degrees of freedom were corrected for violations in the assumption of sphericity using the Greenhouse-Geisser adjustment. 
2.2.1 Accommodative lag. Overall, there was no significant correlation between the separation in component chromaticities of the gratings and the mean accommodative lag (Pearson's $\left.r_{19}=-0.05, p=0.830\right)$, or the standard deviation in the accommodative lag $\left(r_{19}=-0.25\right.$, $p=0.273$ ) (figure 2).

A repeated-measures analysis of covariance with type of grating as factor and colour separation as covariate showed no significant effect of colour separation on the accommodative lag $\left(F_{1,53}=0.04, p=0.846\right)$ and no effect of the grating type (red-blue, blue-green, and green-red) $\left(F_{2,306}=0.03, p=0.968\right)$, or an interaction $\left(F_{2,306}=1.46, p=0.234\right)$.

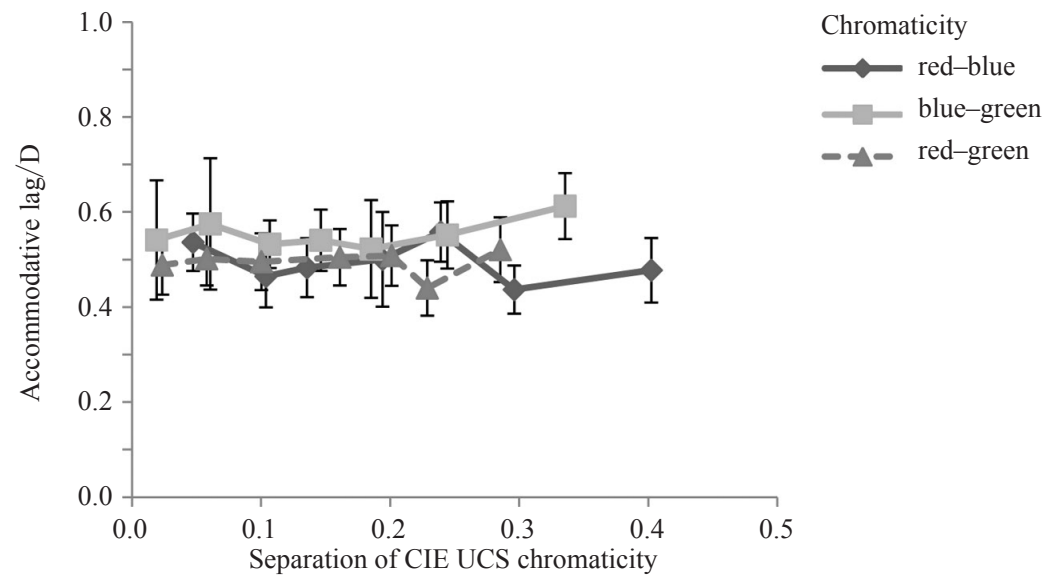

Figure 2. On the left, the accommodative lag for each pattern is shown as a function of the colour difference, separately for the three types of pattern (red-blue, blue-green, and green-red). The larger numerical value for lag indicates a greater accommodative error. Error bars represent 1 SE.

A separate analysis of covariance of the standard deviation in the lag in response to each grating showed that there was no effect of the colour separation $\left(F_{1,153}=0.90, p=0.344\right)$, no effect of the colour pair $\left(F_{1.2,183.4}=3.23, p=0.066\right)$, or an interaction $\left(F_{2,306}=1.37\right.$, $p=0.255)$.

2.2.2 Pupil response. The pupil diameter was not significantly related to the type of pattern or the separation in chromaticity of its bars $\left(r_{19}=-0.10, p=0.672\right)$ (figure 3 ).

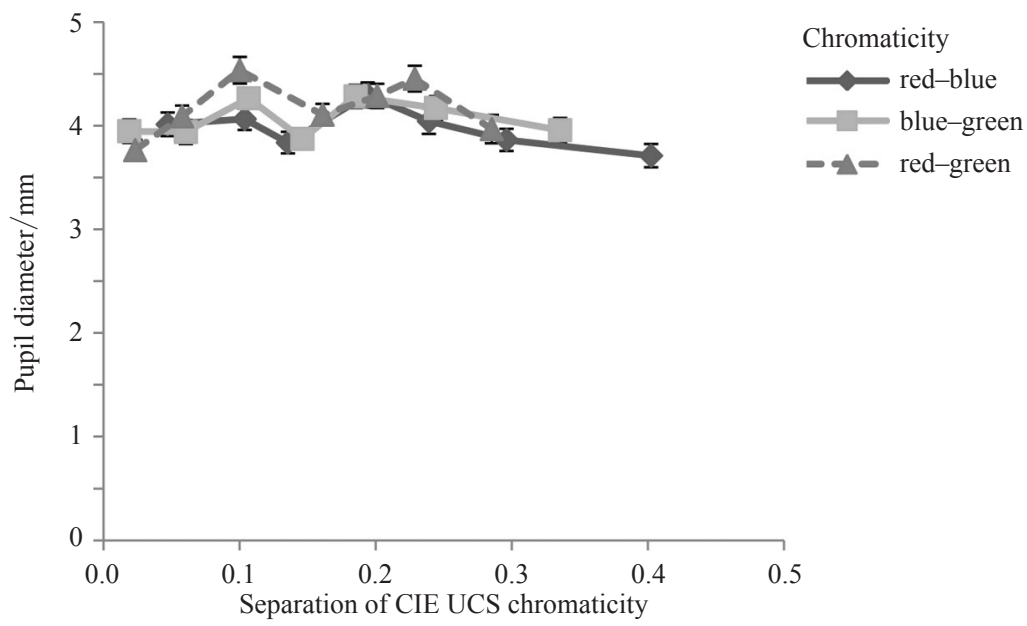

Figure 3. The mean pupil diameter for each pattern is shown as a function of the colour difference, separately for the three types of pattern (red-blue, blue-green, and green-red). The error bars represent $1 \mathrm{SE}$. 


\subsection{Interim discussion}

There was no effect of the pattern on accommodative lag, despite a clear effect of the colour difference on discomfort (Haigh et al 2012b; Wilkins et al 2008). One possible explanation is that the central fixation cross produced an appropriate luminance contrast for accommodation, which negated any effect of the colour difference on accommodation.

In experiment 2 we increased the luminance contrast of the pattern. We used the same procedure as experiment 1 , but the patterns were red-black, green-black, or blue-black, and contained the same central fixation cross. Different wavelengths require different dioptric power due to longitudinal chromatic aberration (LCA). A red object requires more dioptric power than a blue object. There is a neurological blur-reducing mechanism that controls the accommodative system (Phillips and Stark 1977), which indicates that the accommodative response to a red object should be much stronger than the accommodative response to a blue object.

If the results from experiment 1 were due to the fixation cross, then there should be no difference in the lag between the three patterns. If the fixation cross did not affect accommodation, then the red-black pattern should produce the smallest lag, and the blue-black pattern should produce the largest lag due to LCA.

\section{Experiment 2. Effect of LCA on accommodation}

\subsection{Method}

3.1.1 Participants and stimuli. One male and six females from the University of Essex took part, two of whom participated in experiment 1 . The gratings comprised only red pixels, green pixels, or blue pixels, with a space-averaged luminance of $23 \mathrm{~cd} \mathrm{~m}^{-2}$ and dimensions similar to those used in experiment 1.

\subsection{Results}

A repeated-measures analysis of variance showed that there was a main effect of the colour of the grating (red-black, green-black, or blue-black) on the accommodative lag $\left(F_{2,6}=5.20\right.$, $p=0.049$ ). The lag decreased with increasing wavelength as expected from LCA(Friedman's rank test $-\chi_{2}^{2}=6.00, p=0.050$ ) (table 1$)$. There was also a main effect of the colour of the grating on the standard deviation in the lag $\left(F_{2,6}=9.21, p=0.015\right)$, but this was not related to accommodative demand. Friedman's rank test $\left(\chi_{2}^{2}=3.71, p=0.156\right)$.

Table 1. Mean and standard deviation of the accommodative lag from the red-black, green-black, and blue-black gratings.

\begin{tabular}{lll}
\hline Grating & Mean/D & Standard deviation/D \\
\hline Red-black & 0.70 & 0.06 \\
Green-black & 0.72 & 0.05 \\
Blue-black & 0.76 & 0.05 \\
\hline
\end{tabular}

\subsection{Interim discussion}

There was an effect of LCA on the accommodative lag and the standard deviation in the lag despite the presence of the fixation cross. The effect size of the colour-black gratings (experiment 2) was much larger $\left(\eta_{\mathrm{p}}^{2}=0.634\right.$ mean lag; $\eta_{\mathrm{p}}^{2}=0.754$ standard deviation of lag) than the effect size of the gratings that varied in their colour separation (experiment 1) $\left(\eta_{\mathrm{p}}^{2}<0.001\right.$ mean lag; $\eta_{\mathrm{p}}^{2}=0.021$ standard deviation of lag). This suggests that the procedure and instrumentation used in experiment 1 were sensitive enough to detect changes in accommodative lag, if there were changes to be found. Evidently the findings in experiment 1 were not due to the fixation cross and the chromatic separation of the bars of the grating did not affect accommodative lag. 
If the effects of colour on accommodative lag are attributable to LCA, then one might anticipate an effect of LCA on the accommodative response to the large chromaticity separations. The larger the chromaticity separation, the larger the difference in dioptric power needed to de-blur the two bars of the grating. If the accommodative response switches from accommodating to one bar and then accommodating to the other, the large chromaticity separations should have produced a greater variance in the lag. There were, however, no significant effects of chromaticity separation on the variance in the lag detected in experiment 1 or in experiment 2.

Atchison et al (2004) compared the variance in the accommodative response to achromatic images and 'multichromatic' images (chromatic Maltese cross on a coloured background). There was no significant difference between the variance in the accommodative response to the multichromatic images and the variance in the response to the achromatic images. The authors suggest that the accommodative system 'favours' accommodating to shorter wavelengths (larger accommodative lags) more than longer wavelengths (smaller accommodative lags).

In experiment 1 , we also found no effect of LCA on the variance in the accommodative lag. This supports the findings of Atchison et al (2004) who showed that the accommodative response does not switch between accommodating to one colour and then another.

In experiment 1 , there appeared to be no differences in accommodative lag between stimuli. This suggests that the aversion to the large colour differences (Haigh et al 2012b; Wilkins et al 2008) was not due to a larger accommodative lag or greater variance in the lag.

In the next experiment, the gratings differed with respect to movement. Haigh et al (2012a) reported that aversion from moving gratings was greater than aversion from a static grating. The gratings used here were similar to the gratings used by Haigh et al (2012a); therefore, we can be confident that the moving gratings were more aversive than the static grating. Once again there was no relationship between discomfort and accommodative response.

\section{Experiment 3. Accommodation to moving gratings}

Grating patterns that evoke photoparoxysmal responses in the electroencephalograph in patients with photosensitive epilepsy are generally uncomfortable to view (Wilkins et al 1984). Gratings that drift at a constant velocity are not epileptogenic, but gratings that drift at the same velocity but reverse direction (creating a vibrating motion) are highly epileptogenic (Binnie et al 1985). Haigh et al (2012a) reported differences in the contrast thresholds at which drifting, vibrating, and static gratings became aversive. The drifting and vibrating gratings were more uncomfortable to view than static patterns. In particular, the drifting gratings (that are not epileptogenic) were perceived to be more uncomfortable than the (highly epileptogenic) vibrating gratings.

One possible explanation for the discrepancy between the gratings that are epileptogenic and those that are aversive is that the accommodative system is unable to accommodate to images that drift at a constant velocity. If this is the case, then we would expect to see a larger accommodative lag, and/or more variance in the lag, in response to the drifting pattern compared to the static pattern.

The results from experiment 1 show that there was no effect of the uncomfortable patterns on the accommodative response. If the accommodative response is not responsible for the discomfort from grating patterns, no differences in the lag or the variance in the lag in response to the drifting, vibrating, and static patterns would be anticipated.

The accommodative response to the drifting, vibrating, and static patterns was measured. The parameters of movements were such as to increase discomfort. 


\subsection{Methods}

4.1.1 Participants. Four males and twenty-seven females from the University of Essex took part. Twenty-nine of these participants also took part in experiment 1. All participants fulfilled the same criteria as in experiment 1. Participants were asked to complete a questionnaire about their general health, visual history, and any headaches they experienced. None of the participants had a history of seizures.

4.1.2 Stimuli. Horizontal achromatic gratings were created in MATLAB using the PsychToolbox extension (Brainard 1997; Kleiner et al 2007; Pelli 1997) with a square-wave luminance profile and a spatial frequency of $2 \mathrm{cpd}$. One of the gratings was made to drift in a vertical direction orthogonal to the grating, at a constant contour velocity of 10 cycles s$~^{-1}$. The second of the gratings had a similar drifting motion, but the movement abruptly reversed direction after one half spatial cycle to create a vibrating motion. The movement was symmetrical above and below fixation to prevent optokinetic nystagmus; ie the movement was downward in the upper visual field and upward in the lower. The third grating remained static. The gratings were vignetted using a Gaussian window to reduce edge effects. At half contrast the grating subtended $5.6 \mathrm{deg}$. A central fixation cross was superimposed on the gratings $(3 \mathrm{~mm})$. A uniform grey field $\left(54 \mathrm{~cd} \mathrm{~m}^{-2}\right)$ with a central fixation cross appeared between each grating presentation.

4.1.3 Procedure. Each grating was presented for $10 \mathrm{~s}$, followed by $3.5 \mathrm{~s}$ of grey screen. The central fixation cross $(+)$ as replaced by a black square (side $3 \mathrm{~mm}$ ) $0.5 \mathrm{~s}$ before the grating was displayed. The gratings were presented in the following order: drifting (D), vibrating (V), stationary (S), S, V, D, S, D, V, V, D, S, V, S, D, S, V, D. Otherwise, the procedure was the same as in experiment 1.

\subsubsection{Data analysis. The data were analysed as in experiment 1.}

\subsection{Results}

The data from four participants were rejected due the autorefractor being unable to obtain a reading for all of the stimuli, because of excessive blinking or small pupil diameter. As each grating pattern was presented 6 times, the repetition of the presentations was used as an independent variable in the following analyses of variance.

4.2.1 Accommodative response. A repeated-measures analysis of variance was used to test for differences in the mean (table 2) and standard deviation of the accommodative response to the three types of grating: drifting, stationary, and vibrating. There were no significant differences between the mean $\left(F_{1.5,36.7}=2.10, p=0.133\right)$ or the standard deviation $\left(F_{2,50}=0.47, p=0.625\right)$ of the accommodative lag to the gratings.

Table 2. Mean and standard deviation of the accommodative lag to the drifting, vibrating, and static grating patterns.

\begin{tabular}{lll}
\hline Grating type & Mean/D & Standard deviation/D \\
\hline Drifting & 0.50 & 0.12 \\
Vibrating & 0.54 & 0.11 \\
Static & 0.50 & 0.12 \\
\hline
\end{tabular}

4.2.2 Pupil response. There was no effect of the stimulus type on pupil diameter $\left(F_{2,50}=0.23\right.$, $p=0.793)$. 


\subsection{Interim discussion}

There was no effect of the motion of the grating on the accommodative lag or the variance in the lag, indicating that the discomfort from the moving patterns was not due to a larger accommodative lag or more variance in the lag.

Both experiments 1 and 3 failed to find any association between accommodative lag and the ratings that evoke discomfort. It is therefore difficult to attribute the discomfort to accommodative mechanisms; at least this is the case at the viewing distance used. In the next experiment, we looked at the differences in accommodation to static achromatic patterns, and manipulated the perceived viewing distance and thus the accommodative demand. The patterns varied in their spatial frequency and contrast.

\section{Experiment 4. Accommodation to gratings with different spatial frequencies}

Near viewing distances place a greater demand on the accommodative system and produce larger lags of accommodation (Morgan 1968) and more variance in the lag (Jaschinski 1997). Tosha et al (2009) found that participants with high visual discomfort showed significant signs of fatigue when the distance of the target was $4 \mathrm{D}$ compared to $2 \mathrm{D}$. Chase et al (2009) also found larger lags in those with high visual discomfort when viewing targets at 4 D-5 D. If visual discomfort is associated with problems accommodating to the target, then increasing the accommodative demand should emphasise any differences in the size of the lag between the patterns that evoke discomfort and those that do not.

In this experiment, participants were shown 4 grating patterns that varied in their spatial frequency and contrast (see figure 4). The high-contrast, mid-range spatial frequency patterns are within the epileptogenic range and are the most uncomfortable to view (Wilkins et al 1984), so if accommodation is responsible for or related to aversion, we would expect a larger accommodative lag to these patterns. Any effect of the uncomfortable pattern on accommodation should be more apparent when the accommodative demand is greater.

\subsection{Method}

5.1.1 Participants. Seventeen females and twenty males from the Leibniz Research Centre for Working Environment and Human Factors (IfADo), Dortmund, Germany participated. Two participants who habitually wore spectacles did so during the study.

5.1.2 Stimuli. A horizontal achromatic grating was created and presented using PowerPoint on an 8 inch CTF846-A LED monitor (CarTFT.com, Reutlingen, Germany) powered by a Dell Precision M4500 laptop. A 5 D Badal lens was used monocularly to retain the spatial frequency of the grating at various viewing distances. We wished to present monocular accommodative stimuli of constant angular size and identical spatial frequency as a function of viewing distance, ie accommodative load. This can be achieved with the Badal optometer principle (Badal 1876): the eye (ie the corneal apex) was placed at the back focal point of a $5 \mathrm{D}$ (Badal) lens, so that the accommodative load (in dioptres) was proportional to the distance of the target from the front focal point of the lens (Atchison et al 1985): if the target was $20 \mathrm{~cm}$ behind the lens, it appeared at optical infinity.

4 achromatic grating patterns were used. All had a square-wave luminance profile and the stripes had a horizontal orientation. All gratings were circular in outline, subtended $8.5 \mathrm{deg}$ visual angle, and were surrounded by a grey field of $60 \mathrm{~cd} \mathrm{~m}^{-2}$ space-averaged luminance. A grey fixation dot was superimposed on the grating subtending $0.3 \mathrm{deg}$.

The first grating pattern with a spatial frequency of $2 \mathrm{cpd}$ was displayed at maximum contrast $(>95 \%)$ and had a space-averaged luminance of $160 \mathrm{~cd} \mathrm{~m}^{-2}$. For comparison with this aversive pattern, we used a version with the same spatial frequency, but with reduced contrast $(60 \%)$ and reduced space-averaged luminance $\left(85 \mathrm{~cd} \mathrm{~m}^{-2}\right)$. Given the contribution 
of contrast and luminance to aversion, this pattern should have been less aversive. The third and fourth patterns had a contrast of $>95 \%$ and a space-averaged luminance of $160 \mathrm{~cd} \mathrm{~m}^{-2}$, but one had a spatial frequency of $0.25 \mathrm{cpd}$ and the other had a spatial frequency of $3.5 \mathrm{cpd}$ (figure 4). The first and fourth patterns had a spatial frequency close to $3 \mathrm{cpd}$ and the contrast and luminance were high and should therefore have been aversive (Wilkins et al 1984). The second pattern had a lower contrast and luminance and should therefore have been less aversive. The third pattern had a spatial frequency outside the range that induces distortions and discomfort (Wilkins 1995). The four patterns were presented in the order given above.

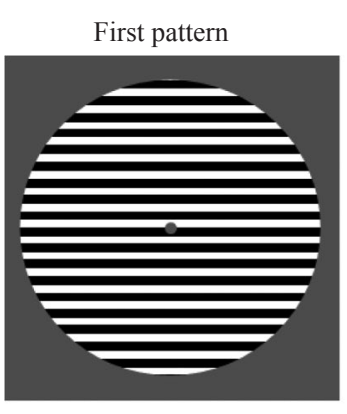

$2 \mathrm{cpd}$ Max contrast

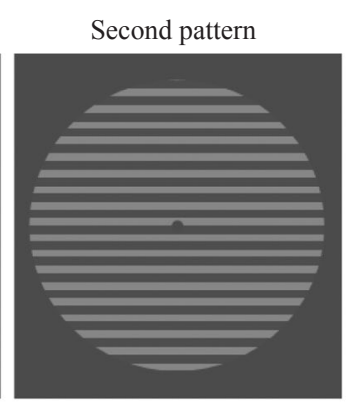

$2 \mathrm{cpd}$ $60 \%$ contrast

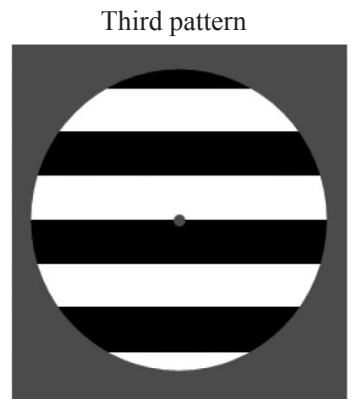

$0.5 \mathrm{cpd}$ Max contrast

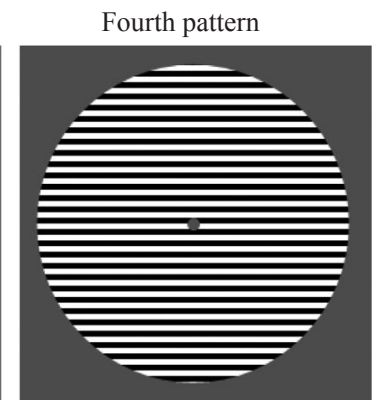

$3.5 \mathrm{cpd}$ Max contrast

Figure 4. Schematics of the 4 grating patterns used in experiment 4.

\subsubsection{Procedure}

Participants viewed each pattern monocularly, first with one eye and then the other, whilst the accommodative response of the viewing eye was measured with an SRW-5000 Autorefractor (Shin-Nippon Commerce Inc, Tokyo, Japan). The patterns were viewed with the left or the right eye first in a counterbalanced order.

For each distance, the participants were asked to "fixieren" (focus on) the central grey fixation dot, whilst single recordings of accommodation were taken (mean 14.5 recordings) over a period of $10 \mathrm{~s}$. When participants were viewing the gratings monocularly, the other eye was occluded by black cloth which hung from the forehead rest on the autorefractor. The Badal lens was placed $200 \mathrm{~mm}$ from the viewing eye of the participant. In terms of the accommodative response required, the $200 \mathrm{~mm}$ distance from the target stimulus to the lens equated to infinity $(0 \mathrm{D})$, the $160 \mathrm{~mm}$ distance to $1 \mathrm{~m}(1 \mathrm{D})$, the $120 \mathrm{~mm}$ distance to $0.5 \mathrm{~m}$ (2 D), the $80 \mathrm{~mm}$ distance to $0.33 \mathrm{~m} \mathrm{(3} \mathrm{D),} \mathrm{and} \mathrm{the} 40 \mathrm{~mm}$ distance to $0.25 \mathrm{~m}(4 \mathrm{D})$.

\subsection{Results}

The monocular recordings from the left and the right eye were analysed separately, so each participant contributed two recordings to each condition. The accommodative response was converted into accommodative lag by subtracting the accommodative response from the accommodative distance. The lag to the target at different distances (1 D-4 D) for each stimulus, and for each eye, was subjected to a regression analysis. The average slope for each participant was then calculated over the range of 1 D-4 D. Tonic accommodation, which is the resting focal distance, is between $1 \mathrm{D}-1.5 \mathrm{D}$ (Leibowitz and Owens 1978). Analysing the slope of the lag-versus-demand curve allows for greater sensitivity when measuring the effect of a stimulus over various distances.

A large lag and/or a steep lag-versus-demand curve indicates a weak response. This can occur because no vergence or proximal stimuli for accommodation were present. To enhance the sensitivity of the measure, slopes that had a gradient greater than 0.6 were rejected from the analysis. 
In addition, two participants had a negative lag in response to the $1 \mathrm{D}$ target consistent with myopia. Following these criteria $(>0.6$ lag-versus-demand slope, and evidence of myopia), six participants' data were excluded from the analysis. Figure 5 shows the average lag-versus-demand curves for the participants who were included and excluded from the analysis. Note, that including the four participants with slopes that were greater than 0.6 did not affect the findings but did add noise to the results.

The mean and the standard deviation of the accommodative lag was analysed as well as the slope of the lag-versus-demand curve for the different distances of the stimulus.

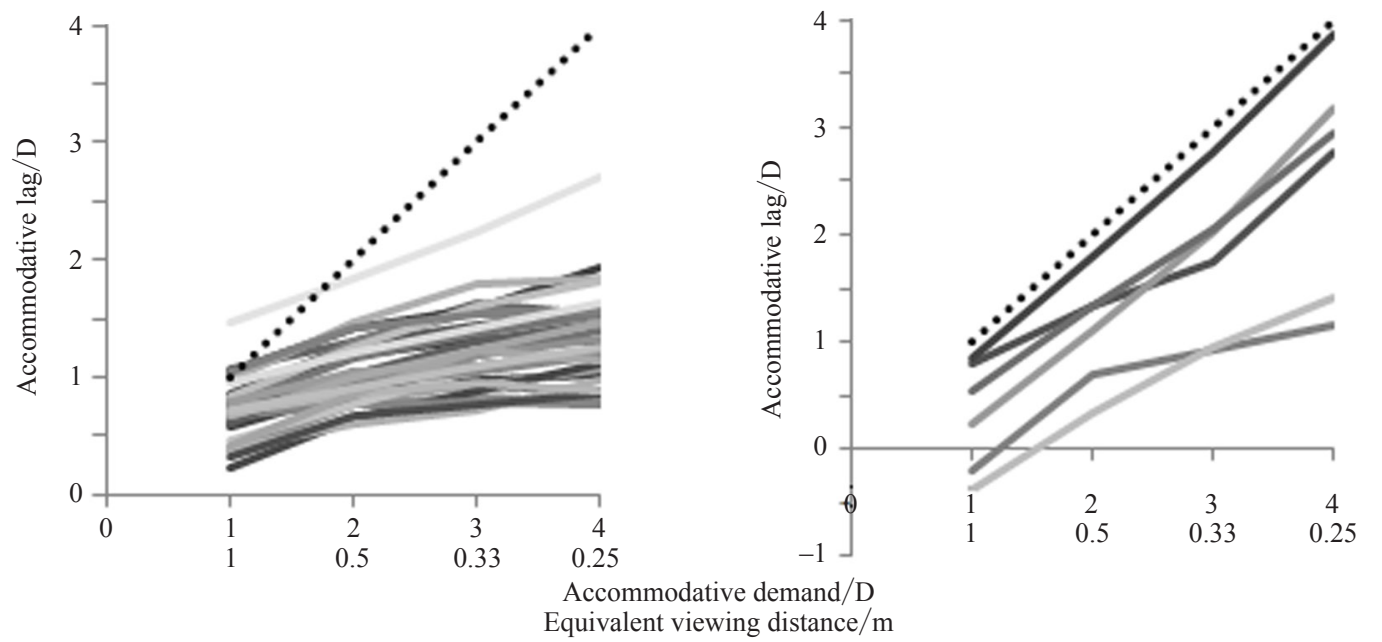

Figure 5. The mean accommodative lag as a function of accommodative demand (equivalent viewing distance) in observers who have been included (left) or excluded (right) from the analysis. The larger the lag, the larger the error in the response. The dotted line shows a slope of 1 (weak accommodative response/large accommodative lag).

5.2.1 Mean accommodative lag. To ensure that there was an effect of the distance of the stimulus on the accommodative lag, a repeated-measures analysis of variance was conducted. The responses were averaged across stimuli for each distance. There was a significant effect of the accommodative demand $\left(F_{1.4,42.5}=134.81, p<0.001\right)$, showing that the near distances created a greater accommodative lag (figure 6).

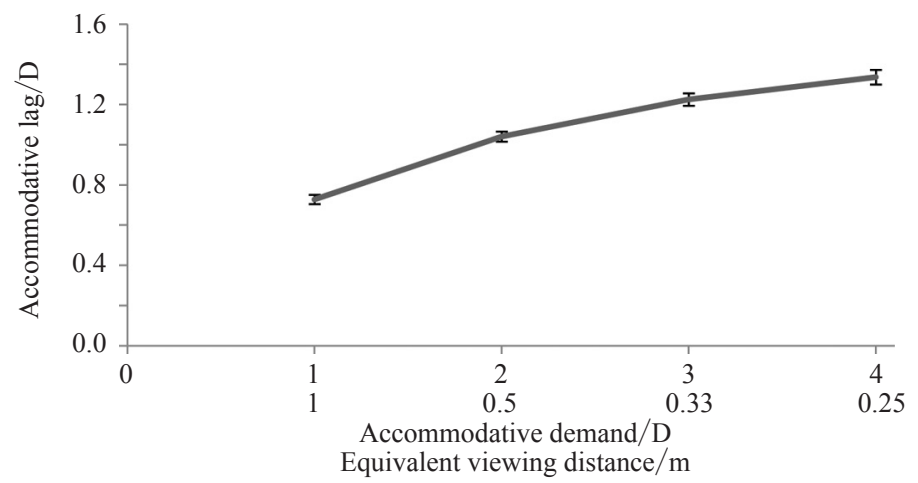

Figure 6. Accommodative lag as a function of accommodative demand. Error bars show 1 SE.

A paired-samples $t$-test showed that there was no significant difference in the slope of the lag-versus-demand curve between the high- and the low-contrast gratings $\left(t_{60}=1.83\right.$, $p=0.072$ ). 
A repeated-measures analysis of variance of the slope of the lag-versus-demand curve to the 3.5, 2, and $0.25 \mathrm{cpd}$ gratings showed that there was no effect of the spatial frequency $\left(F_{1.7,103.2}=2.91, p=0.067\right)$ on accommodation.

The accommodative error index (AEI) assesses the deviation between the slope of the accommodative response and the slope of the perfect accommodative response (Chauhan and Charman 1995). The larger the AEI value, the greater the error in the response. The AEI was calculated for the slope from each participant in response to each of the 4 gratings. There was no significant difference between the high- and low-contrast gratings $\left(t_{60}=1.08\right.$, $p=0.284)$, and no effect of the spatial frequency of the grating $\left(F_{1,60.2}=0.97, p=0.381\right)$.

5.2.2 Variance in accommodative response. The standard deviation of the accommodative response was analysed similarly for all stimuli. There was an overall effect of the viewing distance on the variance in the accommodative response $\left(F_{2.6,160.1}=56.89, p<0.001\right)$, with the accommodation at nearer distances having a greater standard deviation (figure 7 ).

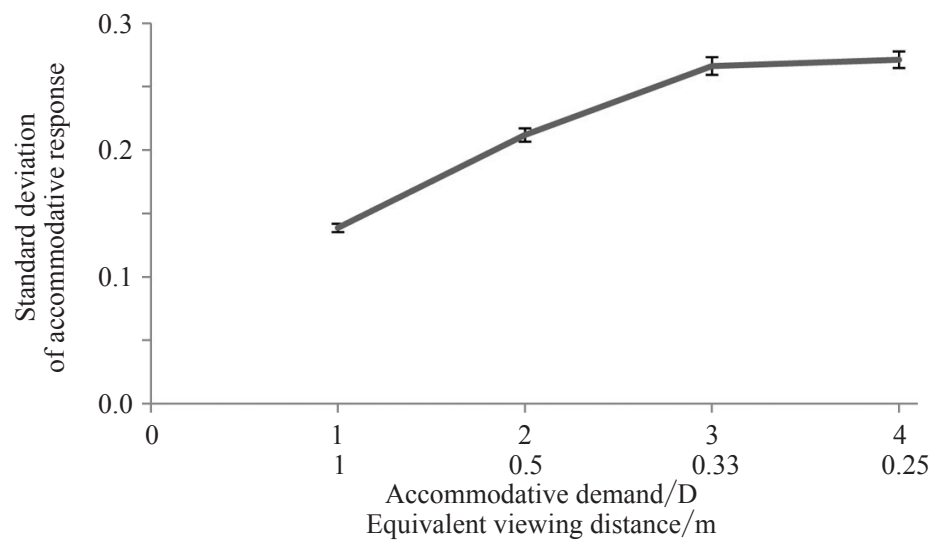

Figure 7. The standard deviation in the accommodative lag as a function of accommodative demand. Error bars represent $1 \mathrm{SE}$.

To analyse the effect of the gratings on the standard deviation of the accommodative lag, analyses of covariance were used, with the accommodative demand as the covariate. When comparing the effect of high- and low-contrast gratings on the standard deviation in the lag, there was a main effect of the covariate $\left(F_{1,250}=21.47, p<0.001\right)$, but there was no effect of the grating $\left(F_{1,250}=0.28, p=0.597\right)$, and no interaction $\left(F_{1,250}=0.01, p=0.918\right)$. Similarly, there was no effect of the spatial frequency of the grating $\left(F_{2,486}=0.17, p=0.840\right)$, and no interaction with the covariate $\left(F_{2,486}=0.12, p=0.891\right)$.

\subsection{Interim discussion}

Once again, there was no effect of the patterns that evoke discomfort on accommodative lag or on the variance in the lag. This was even the case in the $4 \mathrm{D}$ condition. Previous studies found that the differences in accommodative lag between the high and low visual discomfort groups were largest when the distance of the target was $4 \mathrm{D}-5 \mathrm{D}$. Therefore, if there was an effect of the uncomfortable patterns to be found then this should have been evident in the $4 \mathrm{D}$ condition.

It is possible that a longer viewing duration was needed to be able to detect an effect of the grating on the accommodative response. Tosha et al (2009) found effects of fatigue between individuals with high and low visual discomfort after $60 \mathrm{~s}$ of viewing the stimulus. However, when using the pattern glare test (which measures the number of illusions reported in grating patterns that vary in their spatial frequency), each pattern is only presented to the participant for 3 s (Wilkins and Evans 2001), and yet individuals report discomfort and illusions. 
The $10 \mathrm{~s}$ presentation duration was more than sufficient to induce illusions, and if the illusions were the result of accommodation, the effect should have been evident.

There was an effect of accommodative demand on the lag and on the standard deviation of the lag. This was expected and is in accordance with previous findings (Jaschinski 1997; Morgan 1968). We can therefore be confident that the procedure and the autorefractor used were suitable for detecting differences in accommodation to different targets at a variety of different accommodative distances.

\section{General discussion}

For the isoluminant chromatic grating patterns, the moving achromatic patterns, and the patterns that varied in their spatial frequency and contrast, there are clear differences in the discomfort and distortions experienced when viewing the patterns (Haigh et al 2012a, 2012b; Wilkins et al 1984, 2008). Nevertheless there was no effect of the pattern parameters on the accommodative lag or on its standard deviation. As participants showed an effect of longitudinal chromatic aberration on the accommodative lag, we cannot conclude that the procedure or the equipment were insufficiently sensitive to measure changes in accommodation, had such changes been associated with discomfort and distortions. It is possible that the effect of uncomfortable patterns on accommodation is much smaller than the effect of chromatic aberration, but it would be questionable whether such small changes in accommodation could cause the discomfort from the patterns.

The isoluminant patterns that had the greater chromaticity separation would have given rise to greater longitudinal chromatic aberration. The failure to allow for the aberration by adjustment of accommodation might have given rise to discomfort and distortions reported by Haigh et al (2012b) and Wilkins et al (2008). Such a mechanism would not explain the discomfort from the achromatic patterns, and the absence of any association with accommodative lag or variability in accommodative lag.

Experiment 4 was conducted monocularly, which could have reduced the discomfort from the patterns. However, experiment 1 and experiment 3 were conducted binocularly and there was still no effect of the grating on accommodation.

Several studies have found that individual differences in susceptibility to pattern glare/ visual discomfort are related to accommodative lag (Allen et al 2010; Chase et al 2009; Tosha et al 2009). Individuals who experience discomfort tend to have a greater lag of accommodation. This appears to be at odds with the findings discussed above. If there is no relationship between accommodation and the patterns that evoke discomfort, then there should be no relationship across individuals between overall visual discomfort and accommodation. It is possible that the larger lag is a mechanism to cope with the discomfort, rather than a cause of the discomfort. Alternatively, the individuals who habitually suffer visual discomfort may habitually exhibit a greater accommodative lag than others, with no direct association between the visual causes of the discomfort in the short-term.

There is evidence to suggest that the uncomfortable patterns produce a heightened cortical response, and that the heightened response is seen in migraineurs and in healthy control participants (Coutts et al 2012; Huang et al 2003). When the discomfort is reduced by the use of tinted ophthalmic lenses, the cortical response is 'normalised'; that is, the migraineurs then show a similar cortical response to controls (Coutts et al 2012; Huang et al 2011). The experiments reported here suggest that the heightened cortical response to the uncomfortable patterns is not dependent on poor accommodation.

There is no indication that accommodation is responsible for the discomfort induced by grating patterns. Any individual differences in accommodative lag are unlikely to drive the discomfort. 
Acknowledgments. We thank those in the Individual Visual Performance group at the Leibniz Research Centre for Working Environment and Human Factors for their help and support. Some of this work was supported by the Wellcome Trust (grant 80274 to AJW).

\section{References}

Adjamian P, Holliday I E, Barnes G R, Hillebrand A, Hadjipapas A, Singh K D, 2004 "Induced visual illusions and gamma oscillations in human primary visual cortex" European Journal of Neuroscience 20 587-592

Allen P, Hussain A, Usherwood C, Wilkins A, 2010 "Pattern-related visual stress, chromaticity and accommodation" Investigative Ophthalmology \& Visual Science $516843-6849$

Atchison D A, Bradley A, Thibos L N, Smith G, 1995 "Useful variations of the Badal optometer" Optometry and Vision Science 72 279-284

Atchison D A, Strang N C, Stark L R, 2004 "Dynamic accommodation responses to stationary colored targets" Optometry and Vision Science 81 699-711

Badal A, 1876 "Nouvel optomètre, donnant, à la fois et dans une seule opération, la mesure de la réfraction oculaire et celle de l'acuité visuelle" Annales d'Oculistique 75(Jan.-Feb.) 1-13

Binnie C D, Findlay J, Wilkins A J, 1985 "Mechanisms of epileptogenesis in photosensitive epilepsy implied by the effects of moving patterns" Electroencephalography and Clinical Neurophysiology 61 1-6

Brainard D H, 1997 “The Psychophysics Toolbox" Spatial Vision $10433-436$

Campbell F W, Robson J G, 1958 "Moving visual images produced by regular stationary patterns" Nature 181362

Chase C, Tosha C, Borsting E, Ridder W H I, 2009 "Visual discomfort and objective measures of static accommodation" Optometry and Vision Science 86 883-889

Chauhan K, Charman W N, 1995 "Single figure indices for the steady-state accommodative response" Ophthalmic and Physiological Optics 15 217-221

Coutts L V, Cooper C E, Elwell C E, Wilkins A J, 2012 "Time course of the haemodynamic response to visual stimulation in migraine, measured using near-infrared spectroscopy" Cephalalgia 32 $621-629$

Georgeson M A, 1976 "Psychophysical hallucinations of orientation and spatial frequency" Perception 5 99-111

Georgeson M A, 1980 "The perceived spatial frequency, contrast, and orientation of illusory gratings" Perception 9 695-712

Gregory R L, 1977 "Vision with isoluminant colour contrast: 1. A projection technique and observations" Perception 6 113-119

Haigh S M, Karanovic O, Wilkinson F, Wilkins A J, 2012a "Cortical hyperexcitability in migraine and aversion to patterns" Cephalalgia 32 236-240

Haigh S M, Tang P, Barningham L, Coutts L, Allen P M, Wilkins A J, 2012b “Colour separation and aversion" i-Perception 3232

Helmholtz H, 1856/1924 Helmholtz's Treatise on Physiological Optics volume 1, translated from the third German edition of Handbuch der physiologischen Optik (Leipzig: Voss) by J P C Southall (New York: Optical Society of America)

Huang J, Cooper T, Satana B, Kaufman D, Cao Y, 2003 "Visual distortion provoked by a stimulus in migraine associated with hyperneuronal activity" Headache 43 664-671

Huang J, Zong X, Wilkins A, Jenkins B, Bozoki A, Cao Y, 2011 "fMRI evidence that precision ophthalmic tints reduce cortical hyperactivation in migraine" Cephalalgia 31 925-936

Jaschinski W, 1997 "Fixation disparity and accommodation as a function of viewing distance and prism load" Ophthalmic and Physiological Optics 17 324-339

Kleiner M, Brainard D, Pelli D, 2007 "What's new in Psychtoolbox-3? Perception 36 ECVP Supplement, 14

Leibowitz H W, Owens D A, 1978 "New evidence for the intermediate position of relaxed accommodation" Documenta Ophthalmologica 46 133-147

Marcus D A, Soso M J, 1989 "Migraine and stripe-induced visual discomfort" Archives of Neurology 6 1129-1132 
Millodot M, 1968 "Influence of accommodation on the viewing of an optical illusion" Quarterly Journal of Experimental Psychology 20 329-335

Morgan M W, 1968 "Accommodation and vergence" American Journal of Optometry \& Archives of American Academy of Optometry 45 417-454

Pelli D G, 1997 "The VideoToolbox software for visual psychophysics: Transforming numbers into movies" Spatial Vision 10 437-442

Phillips S, Stark L, 1977 "Blur: A sufficient accommodative stimulus” Documenta Ophthalmologica 43 65-89

Purkinje J (Ed.), 1823 Beobachtungen und Versuche zur Physiologie der Sinne. Beiträge zur Kenntniss des Sehens in subjecktiver Hinsicht volume 1 (Prague: Calve'schen Buchhandlung)

Purkinje J (Ed.), 1825 Beobachtungen und Versuche zur Physiologie der Sinne. Neue Beiträge zur Kentniss des Sehens in subjectiver Hinsicht volume 2 (Berlin: Reiner)

Simmers A J, Gray L S, Wilkins A J, 2001 "The influence of tinted lenses upon ocular accommodation" Vision Research 41 1229-1238

Siniatchkin M, Moeller F, Shepherd A, Siebner H, Stephani U, 2007 "Altered cortical visual processing in individuals with a spreading photoparoxysmal EEG response" European Journal of Neuroscience 26 529-536

Switkes E, Bradley A, Schor C, 1990 "Readily visible changes in color contrast are insufficient to stimulate accommodation" Vision Research 30 1367-1376

Tosha C, Borsting E, Ridder W H, Chase C, 2009 "Accommodation response and visual discomfort" Ophthalmic and Physiological Optics 29 625-633

Wade N J, 1983 Brewster and Wheatstone on Vision" (London: Academic Press)

Wilkins A J, 1986 "What is visual discomfort?" Trends in Neuroscience 9 343-346

Wilkins A J, Evans B J W, 2001 Pattern Glare Test Instructions (London: i.O.O. Sales)

Wilkins A J, Nimmo-Smith M I, 1987 "The clarity and comfort of printed text" Ergonomics 30 $1705-1720$

Wilkins A J, Nimmo-Smith M I, Tait A, McManus C, Della Sala S, Tilley A, Arnold K, Barrie M, Scott S, 1984 "A neurological basis for visual discomfort" Brain 107 989-1017

Wilkins A J, Tang P, Irabor J, Baningham L, Coutts L, 2008 "Cortical haemodynamic response to coloured gratings" Perception 37 ECVP Supplement, 144

Wolfe J M, Owens D A, 1981 "Is accommodation colourblind? Focusing chromatic contours" Perception $1053-62$

Wolffsohn J S, Hunt O A, Gilmartin B, 2002 "Continuous measurement of accommodation in human factor applications” Ophthalmic and Physiological Optics 22 380-384 